\section{Determinantes socioeconómicos de la atención odontológica durante la gestación en Cali, Colombia}

\author{
Socioeconomic determinants of dental care during \\ pregnancy in Cali, Colombia
}

\author{
Determinantes socioeconômicos do atendimento \\ odontológico durante a gravidez em Cali, \\ Colômbia
}




\section{Introducción}

La atención odontológica durante el embarazo es una oportunidad para fortalecer conductas y hábitos de promoción de la salud y prevención de la enfermedad. La detección oportuna de patologías orales, asociadas con compromisos sistémicos en la gestante, puede contribuir a disminuir las complicaciones relacionadas con parto y las consecuencias derivadas del bajo peso al nacer 1,2 .

En una época donde los beneficios de los avances científicos en materia de salud general y en salud bucodental, en particular, no están distribuidos equitativamente, el abordaje de los determinantes sociales de la salud es necesario para orientar a los gobiernos en la reducción de las desigualdades. Las acciones en salud bucodental deben dirigirse por lo tanto no sólo a los factores del comportamiento en la construcción de hábitos saludables, sino a los entornos sociales en los que las conductas de salud se desarrollan ${ }^{3}$.

Diversos estudios muestran desconocimiento en los programas de control prenatal de aspectos relacionados con la salud bucodental y el desconocimiento del personal de odontología de los riesgos sistémicos de las gestantes 4,5,6,7.

Según pruebas científicas, la salud bucodental y la salud general están interrelacionadas, en particular, las infecciones bucodentales y los resultados adversos del embarazo 8,9. Dado el costo social y familiar de estos resultados adversos -como los bebés nacidos con bajo peso, se justifica una acción oportuna de la sociedad mediante políticas públicas encaminadas a mejorar la salud bucodental de las gestantes.

En estudios realizados en países en vía de desarrollo, solamente entre el 30 y el 55\% de las gestantes fueron atendidas en odontología, comparado con un 70 al $90 \%$ en países desarrollados 10 . Esta es una situación preocupante, lo que hace necesario generar estudios que permitan contar con datos que orienten al tomador de decisiones en los niveles locales para mejorar las condiciones de salud bucodental de esta población, que por sus características está expuesta a patologías relacionadas con parto pre término y el bajo peso al nacer.

En Colombia, el sistema de salud incluye planes de beneficios a las gestantes a través de las Empresas Promotoras de Salud (EPS), que son las encargadas del aseguramiento en salud, tanto de la población del régimen contributivo (aquellos que tiene capacidad de pago por ser trabajador dependiente o independiente y sus beneficiarios), como de la población pobre afiliada al régimen subsidiado. Las EPS contratan a su vez los servicios de salud a las Instituciones Prestadoras de Servicios de Salud (IPS) tanto públicas como privadas. La población pobre que aún no está asegurada en salud, recibe atención a través de la red pública de hospitales con recursos del Estado. La mayoría de partos en Cali son atendidos en la red privada, debido a que la mayoría usuarias están afiliadas a EPS del régimen contributivo. De los 28.811 nacimientos estimados en Cali para el 2011,64,3\% fueron atendidos en la red privada y $55 \%$ estaban afiliadas al régimen contributivo (Secretaría de Salud de Cali. Análisis situación salud Cali 2011. http://www.calisaludable.cali. gov.co/proyectos/, accedido el 12/Ene/2012). Cali tenía una población estimada para el año 2012 de 2.194.781 habitantes 11.

Este estudio pretende estimar la prevalencia de la asistencia a la consulta dental de las gestantes atendidas en las principales clínicas públicas y privadas de Cali, Colombia en el año 2012, e identificar factores socioeconómicos y de los servicios de salud asociados a recibir atención odontológica durante el embarazo.

\section{Material y métodos}

Se realizó un estudio transversal, en el que participaron 993 madres en puerperio inmediato que habían sido atendidas en 4 clínicas públicas y 4 privadas de la ciudad de Cali, capital del Departamento Valle del Cauca, Colombia en el transcurso de 2012. Para calcular el tamaño de la muestra $(n=998)$ se utilizó el programa Epidat v3.1 (Xunta de Galicia, España; http://dxsp. sergas.es/default.asp); se consideró una precisión de 0,0366, un nivel de confianza de 95\% y un efecto de diseño de 1,5; se asumió como población número de nacimientos reportados en el informe de gestión de la Secretaría de Salud de Cali de 2010, que fue de 30.42012 . La prevalencia (61.5\%) fue tomada de estudios previos, realizados para el levantamiento de línea base de salud oral en el Valle del Cauca 2009 13; sólo cinco madres rechazaron participar en el estudio. De las 19 clínicas con sala de partos existentes en Cali (14 privadas y 5 públicas), fueron escogidas para el estudio aquellas 8 (4 privadas y 4 públicas) que brindan atención prioritariamente al municipio de Cali y atienden partos de baja y mediana complejidad. Estas 8 clínicas atendieron el $73 \%$ de los partos ocurridos en la ciudad en 2012. A partir de considerar el número de partos diarios promedio por clínica, se eligieron aleatoriamente los días para las entrevistas, teniendo cada clínica igual probabilidad de ser seleccionada diariamente, hasta completar la muestra. En cada día seleccionado, se entrevistó a todas las madres en puerperio inmediato. 
El cuestionario fue diseñado para evaluar las características de la madre distribuidas en cuatro dimensiones: socio-demográficas, servicios de salud, económicas y prácticas en salud oral. El formulario empleado para la recolección de la información fue organizado de acuerdo con las sugerencias planteadas en otros estudios 14,15,16. En la prueba piloto se aplicó el instrumento a 40 madres que se encontraban en puerperio inmediato en cuatro clínicas, donde asisten los partos a usuarias afiliadas del régimen contributivo y del régimen subsidiado del sistema del seguridad social en salud colombiano (2 clínicas atendían régimen contributivo y las otras 2 atendían régimen subsidiado). $73 \%$ de las preguntas no tuvieron modificación; se tuvo en cuenta para la modificación aquellos ítems que no fueron contestados en un porcentaje mayor a $66 \%$ y los reportes del entrevistador y el observador de campo.

\section{Variables}

\section{- Variable dependiente}

La asistencia al servicio de odontología durante el embarazo, la cual es dicotómica, con las opciones sí o no, dependiendo si la gestante asistió o no alguna vez a la consulta odontológica.

\section{- Variables independientes}

En la dimensión sociodemográfica se estudiaron: edad, categorizada como menores de 18 años y 18 años y más; estado marital (en convivencia con pareja o sin pareja); grupo étnico (afrocolombiana, resto de la población); escolaridad (hasta secundaria incompleta, secundaria completa o más) paridad (primípara, multípara). A nivel económico se indagó sobre percepción de ingresos en el hogar (ingresos bajos, ingresos medios o altos), percepción de suficiencia recursos para pago de necesidades básicas (suficiente o justo para vivir, insuficiente), el tipo de aseguramiento en salud (contributivo aquellas con capacidad de pago; subsidiado aquellas clasificadas como población pobre ingresadas al régimen subsidiado, y no aseguradas a aquella población pobre no incluida aún en el régimen subsidiado, pero cubiertos por los hospitales públicos). La dimensión servicios de salud tuvo en cuenta el sitio de atención del control prenatal y sitio de atención dental (red pública, red privada, orientación en el control prenatal sobre acceso y atención en salud oral (sí, no). Las madres que se encontraban en sala de puerperio fueron invitadas a participar del estudio y se les solicitó consentimiento informado, según formato aprobado por los comités de ética de las clínicas participantes.

\section{Análisis estadístico}

La entrada de datos y análisis de los resultados se realizó utilizando el paquete estadístico para ciencias sociales (IBM SPSS versión 18.0; IBM Corp., Armonk, Estados Unidos). Las estadísticas descriptivas como media y desviación estándar (SD) para las variables continuas, y la frecuencia y el porcentaje para las variables categóricas fueron utilizados. La prueba de chi-cuadrado se utilizó para comparar los perfiles de las madres que visitaron dentista durante el embarazo actual, con las que no. El nivel de significación se fijó en 0,05 . Los factores asociados con la asistencia de la madre al servicio de odontología se determinaron, tanto a nivel bivariado, como multivariado mediante análisis de regresión logística y análisis de regresión logística múltiple, respectivamente, calculándose OR (odds ratio) crudos y ajustados y su intervalo de confianza del 95\% (IC95\%). Se asumió que aquellas variables, cuya asociación con la asistencia a consulta odontológica a nivel bivariado fueran estadísticamente significativas, se incorporaran al análisis multivariado.

Las mujeres que se encontraban en sala de puerperio fueron invitadas a participar en el estudio y se les solicitó consentimiento informado, según formato aprobado por los comités de ética de las clínicas participantes.

\section{Resultados}

Se entrevistaron 993 madres, de las cuales el 50\% tenían 23 años o menos y 23,6\% eran adolescentes (gestantes menores de 20 años); la media de la distribución fue 19 años; la edad promedio, 24,7 (IC95\%: 24,3-25,1), con una desviación estándar de 6,1. El 83\% de las entrevistadas (IC95\%: 80,6$85,4)$ asistieron a odontología; las gestantes presentaron las siguientes características agrupadas por dimensiones.

\section{Dimensión sociodemográfica}

La edad no resultó asociada con la asistencia a odontología cuando se compararon todas las edades. Al generar el subgrupo de menores de edad, se encontró que asistieron en menor proporción a la consulta de odontología que el resto de las madres $(76,2 \%$ versus $83,7 \%$; OR $=1,6$; IC95\%: 1,0-2,6; estadístico Fisher < 0,05).

$\mathrm{Al}$ analizar el aspecto étnico se pudo observar que las gestantes mestizas, indígenas y aquellas sin definición de etnia, asistieron a odontología en mayor proporción que las gestantes afrocolombianas. Solamente el $26 \%$ de las madres afrocolombianas estaban afiliadas al régimen 
contributivo, comparado con las no afrocolombianas (había 4,6 gestantes no afrocolombianas afiliadas al régimen contributivo por cada madre afrocolombiana; IC95\%: 3,4-6,4). 60,3\% de las madres no afrocolombianas asistieron a odontología en clínicas privadas versus $39,7 \%$ de las afrocolombianas (OR = 3,5; IC95\%: 2,5-5,0). Las no afrocolombianas habían alcanzado un mayor nivel educativo que las afrocolombianas $(69,8 \%$ versus $30,2 \%$, OR = 2,5; IC95\%: 1,9-3,5).

Las madres que durante su gestación convivían con su pareja asistieron en mayor proporción que aquellas que estaban solas. Cuanto mayor mayor fue el nivel educativo alcanzado por la gestante, mayor era la proporción de que asistiera a odontología. Las madres primíparas asistieron en mayor proporción al odontólogo que las multíparas (Tabla 1). Con relación a la ocupación, 54,5\% de las entrevistadas estaban dedicadas al hogar, $31,3 \%$ trabajaban, $10 \%$ estudiaban y $4,2 \%$ realizaban más de una actividad; la ocupación no estuvo relacionada con la visita de la gestante a odontología.

\section{Dimensión económica}

Según el tipo de seguridad social a la cual estaban afiliadas las entrevistadas, se encontró que el 52,6\% contaban con un carné del régimen contributivo; $36 \%$ manifestaron que eran usuarias del régimen subsidiado y un $11,5 \%$ no estaban aseguradas aún en el sistema de seguridad social. Cerca de la mitad de madres $(48,4 \%)$ comentaron que los recursos con que cuenta son apenas lo justo para vivir. El resto de población manifestó posiciones extremas en este aspecto, una cuarta parte percibe sus ingresos suficientes y la otra cuarta parte los percibe como insuficientes. Los ingresos percibidos en el hogar se distribuyeron según lo manifestado por las madres entre muy bajo y bajo el $29,4 \%$; ingresos medios el $62,1 \%$ y entre medio-alto y relativamente alto el 8,5\%. Quienes expresaron que tenían recursos suficientes, habían manifestado que sus ingresos estaban entre medio-alto y relativamente alto; aquellas que señalaron tener recursos insuficientes, en un $65 \%$ habían manifestado que sus ingresos eran entre muy bajos y bajos; finalmente quienes dijeron que sus recursos les alcanzaban sólo para vivir habían manifestado que sus ingresos eran de nivel medio en un $60 \%(\mathrm{p}<0,001)$.

La forma de pago en el control prenatal de quienes asistieron a odontología fue más frecuente en aquellas que presentaron exclusivamente el carné $(75,1 \%)$ y en aquellas que lo hicieron en forma mixta, es decir, presentando el carné más recursos propios $(24,8 \%),(\mathrm{p}<0,01)$.
Se encontró que las gestantes con mejores condiciones socioeconómicas, medidas a través del tipo de aseguramiento y de la percepción sobre ingresos y suficiencia de recursos, asistieron en mayor porcentaje a odontología (Tabla 2).

\section{Dimensión servicios}

La asistencia al control prenatal de quienes fueron a odontología lo hicieron en las clínicas privadas de las EPS $(48,8 \%)$ y en las instituciones de la red pública $(44,1 \%)$; el resto asistieron al médico particular; sólo un 1,8\% asistieron tanto a clínicas de la red pública como privada. Las que manifestaron haber asistido al control prenatal en la red privada, asistieron a odontología en el $88,8 \%$ y las que asistieron a la red pública asistieron a odontología en un 76,9\% ( $\mathrm{p}<0,01)$.

Las madres que durante su gestación fueron informadas por el control prenatal de asistir a odontología y aquellas que fueron orientadas en cómo cuidar sus dientes, asistieron en mayor proporción que aquellas que no recibieron dicha información. Además, la mayoría de las gestantes que fueron a odontología lo hicieron durante el primer y segundo trimestre $(50,5 \%$ y $30,2 \%$ respectivamente, $\mathrm{p}<0,01$ ). La principal razón de asistencia a odontología fue la remisión (Tabla 3).

Para explorar con mayor certeza posibles hipótesis acerca del acceso de las gestantes al servicio de odontología, y cómo en ello influyen las dimensiones: social, socioeconómica y servicios de salud previamente analizados, se incorporaron al análisis multivariado aquellas variables cuya asociación con la asistencia a consulta odontológica a nivel bivariado fuera estadísticamente significativa; son los casos del estado marital, etnia, nivel de instrucción, tipo de aseguramiento, suficiencia de ingresos para el pago de necesidades básicas, sitio de atención del parto, orientación durante el embarazo en cómo cuidar los dientes, control prenatal suministro información para asistir a odontología y paridad (Tabla 4).

Se encontró que el mayor peso lo tienen las variables relacionadas con los servicios de salud: las madres que contaron con remisión del control prenatal hacia odontología durante el embarazo o que recibieron en el control prenatal orientación en salud oral tuvieron, respectivamente, seis y cinco veces mayor probabilidad de asistir a consulta de odontología que las que no fueron remitidas ni recibieron orientación sobre salud oral, controlando el efecto del resto de las variables incluidas en el análisis. En la dimensión social tuvieron un mayor peso relativo el estado marital -la probabilidad de que una madre con pareja estable asistiera a consulta odontológica casi triplicó la de las madres sin pareja- y la 
Tabla 1

Asociación entre variables sociodemográficas y la asistencia a odontología durante el embarazo. Cali, Colombia, 2012.

\begin{tabular}{|c|c|c|c|c|c|}
\hline Variable medida & $\begin{array}{c}n \\
{[N=993]}\end{array}$ & $\begin{array}{c}\text { Sí asistió (\%) } \\
{[n=824]}\end{array}$ & $\begin{array}{c}\text { No asistió (\%) } \\
{[n=169]}\end{array}$ & OR & IC95\% \\
\hline \multicolumn{6}{|l|}{ Estado marital } \\
\hline Convivencia pareja (casada, unión libre) & 812 & 85,8 & 14,2 & 2,6 & $1,8-3,7$ \\
\hline Sin pareja (soltera, separada, divorciada, viuda) & 181 & 70,2 & 29,8 & 1,0 & \\
\hline \multicolumn{6}{|l|}{ Comparativo } \\
\hline Afrocolombianas & 265 & 75,5 & 24,5 & 1,0 & \\
\hline Resto población & 728 & 85,7 & 14,3 & 2,0 & $1,4-2,8$ \\
\hline \multicolumn{6}{|l|}{ Escolaridad } \\
\hline Secundaria completa o técnica & 570 & 87,4 & 12,6 & & \\
\hline Secundaria incompleta & 268 & 77,2 & 22,8 & & \\
\hline Primaria & 87 & 63,2 & 36,8 & & \\
\hline Superior & 65 & 93,8 & 6,2 & & \\
\hline Ninguna & 3 & 100,0 & 0,0 & & \\
\hline \multicolumn{6}{|l|}{ Nivel de instrucción } \\
\hline Hasta secundaria incompleta & 358 & 74,0 & 26,0 & 1,0 & \\
\hline Secundaria completa y superior & 635 & 88,0 & 12,0 & 2,6 & $1,8-3,6$ \\
\hline \multicolumn{6}{|l|}{ Comparativo menores de 18 años edad vs. resto } \\
\hline Menor de 18 años & 101 & 76,2 & 23,8 & 1.0 & \\
\hline 18 años o más & 892 & 83,7 & 16,3 & 1,6 & $1,0-2,6$ \\
\hline \multicolumn{6}{|l|}{ Paridad } \\
\hline Primípara & 519 & 87,9 & 12,1 & 2,1 & $1,5-2,9$ \\
\hline Multípara & 474 & 77,6 & 22,4 & 1,0 & \\
\hline
\end{tabular}

IC95\%: intervalo de confianza del 95\%; OR: odds ratio.

Tabla 2

Asociación entre variables económicas y la asistencia a odontología durante el embarazo. Cali, Colombia, 2012.

\begin{tabular}{|c|c|c|c|c|c|}
\hline Variable medida & $\begin{array}{c}n \\
{[N=993]}\end{array}$ & $\begin{array}{c}\text { Sí asistió (\%) } \\
{[n=824]}\end{array}$ & $\begin{array}{c}\text { No asistió (\%) } \\
{[n=169]}\end{array}$ & OR & $\mathrm{IC} 95 \%$ \\
\hline \multicolumn{6}{|l|}{ Régimen de seguridad social } \\
\hline Contributivo & 522 & 88,7 & 11,3 & & \\
\hline Subsidiado & 357 & 76,2 & 23,8 & & \\
\hline No aseguradas & 114 & 78,1 & 21,9 & & \\
\hline \multicolumn{6}{|l|}{ Comparativo } \\
\hline Régimen contributivo & 522 & 88,7 & 11,3 & 2,4 & $1,7-3,4$ \\
\hline Régimen subsidiado y no aseguradas & 471 & 76,6 & 23,4 & & \\
\hline \multicolumn{6}{|l|}{ Suficiencia-insuficiencia } \\
\hline Suficiente o justo para vivir & 738 & 86,6 & 13,4 & 2,4 & $1,7-3,5$ \\
\hline Insuficientes & 255 & 72,5 & 27,5 & & \\
\hline \multicolumn{6}{|l|}{ Comparativo ingresos bajo-no bajo } \\
\hline Ingresos bajos & 292 & 73,3 & 26,7 & & \\
\hline Ingresos medios o altos & 701 & 87,0 & 13,0 & 2,4 & $1,7-3,4$ \\
\hline
\end{tabular}

IC95\%: intervalo de confianza del 95\%; OR: odds ratio. 
Tabla 3

Asociación entre variables del servicio de salud y la asistencia a odontología durante el embarazo. Cali, Colombia, 2012.

\begin{tabular}{|c|c|c|c|c|c|}
\hline Variable medida & $\begin{array}{c}n \\
{[N=993]}\end{array}$ & $\begin{array}{l}\text { Sí asistió (\%) } \\
\text { [n = 824] }\end{array}$ & $\begin{array}{c}\text { No asistió (\%) } \\
{[n=169]}\end{array}$ & OR & $\mathrm{IC} 95 \%$ \\
\hline Sitio de atención & 993 & 83,0 & 17,0 & & \\
\hline Parto clínica red pública & 393 & 76,3 & 23,7 & 1,0 & \\
\hline Parto clínica red privada & 600 & 87,3 & 12,7 & 2,1 & $1,5-3,0$ \\
\hline Control prenatal clínicas red pública & 438 & 76,9 & 23,1 & 1,0 & \\
\hline Control prenatal clínicas red privada & 528 & 88,8 & 11,2 & 2,4 & $1,7-3,4$ \\
\hline \multicolumn{6}{|c|}{ Recibió en el control prenatal información para asistir a odontología } \\
\hline Sí & 916 & 86,4 & 13,6 & 8,4 & $5,2-13,8$ \\
\hline No & 77 & 42,9 & 57,1 & 1,0 & \\
\hline \multicolumn{6}{|c|}{ Le orientaron cómo cuidar los dientes durante embarazo } \\
\hline Sí & 689 & 91,0 & 9,0 & 5,5 & $3,9-7,8$ \\
\hline No & 304 & 64,8 & 35,2 & 1,0 & \\
\hline \multicolumn{6}{|l|}{ Comparativo remisión versus otras } \\
\hline Por remisión & 424 & 95,8 & 4,2 & 8,1 & $4,9-13,5$ \\
\hline Por otras razones & 569 & 73,5 & 26,5 & 1,0 & \\
\hline
\end{tabular}

IC95\%: intervalo de confianza del 95\%; OR: odds ratio.

Tabla 4

Asociación de variables sociodemográficas, económicas y de servicios con la asistencia a odontología durante el embarazo: resultados del análisis multivariado. Cali, Colombia, 2012.

\begin{tabular}{|c|c|c|c|c|}
\hline Variable medida & OR crudo & IC95\% & OR ajustado & IC95\% \\
\hline \multicolumn{5}{|l|}{ Estado marital } \\
\hline Convivencia pareja (casada, unión libre) & 2,6 & $1,8-3,7$ & 2,8 & $1,7-4,5$ \\
\hline Sin pareja (soltera, separada, divorciada, viuda) & 1,0 & & 1,0 & \\
\hline \multicolumn{5}{|l|}{ Nivel de instrucción } \\
\hline Hasta secundaria incompleta & 1,0 & & 1,0 & \\
\hline Secundaria completa y superior & 2,6 & $1,8-3,6$ & 1,7 & $1,1-2,7$ \\
\hline \multicolumn{5}{|c|}{ Suficiencia de ingresos para pago de necesidades básicas } \\
\hline Suficiente o lo justo para vivir & 2,4 & $1,7-2,4$ & 1,8 & $1,1-2,8$ \\
\hline Insuficientes & 1,0 & & 1,0 & \\
\hline \multicolumn{5}{|l|}{ Sitio de atención } \\
\hline Clínica red pública & 1,0 & & 1,0 & \\
\hline Clínica red privada & 2,1 & $1,5-3,0$ & 2,3 & $1,4-3,7$ \\
\hline \multicolumn{5}{|c|}{ Le orientaron cómo cuidar sus dientes durante el embarazo } \\
\hline Sí & 1,0 & & 1,0 & \\
\hline No & 5,5 & $3,9-7,8$ & 6,0 & $3,9-9,2$ \\
\hline \multicolumn{5}{|c|}{ Recibió en el control prenatal información para asistir a odontología } \\
\hline Sí & 1,0 & & 1,0 & \\
\hline No & 8,4 & $5,2-13,8$ & 5,7 & $3,1-10,8$ \\
\hline \multicolumn{5}{|l|}{ Comparativo remisión vs otras causas de consulta } \\
\hline Por remisión & 8,1 & $4,9-13,5$ & 7,0 & $4,0-12,0$ \\
\hline Por otras razones & 1,0 & & 1,0 & \\
\hline \multicolumn{5}{|l|}{ Paridad } \\
\hline Primípara & 2,1 & $1,5-2.9$ & 2,5 & $1,6-3,8$ \\
\hline Multípara & 1,0 & & 1,0 & \\
\hline
\end{tabular}

IC95\%: intervalo de confianza del 95\%; OR: odds ratio. 
paridad, pues la probabilidad de que una madre primípara hubiera asistido durante el embarazo a la consulta dental más que duplicó a la de las madres primíparas; en lo socioeconómico, el que la madre percibiera que satisfacía adecuadamente sus necesidades básicas en el hogar tuvo una fuerte asociación con la asistencia a consulta odontológica durante la gestación.

En general, los OR estimados en el análisis multivariado no difieren sustancialmente de los encontrados en el análisis bivariado, salvo en el caso de las gestantes que recibieron en el control prenatal orientación en salud oral, factor en el que se observa una notable reducción en el valor del OR (de 8,4 a 5,7).

El perfil socioeconómico de las madres que durante el embarazo no asistieron a odontología correspondió en dos terceras partes a población que puede ser considerada en condiciones de pobreza. Entre las razones dadas por quienes no asistieron a odontología, un 18,3\% consideró que no era necesario ir, un $17,2 \%$ no tenía tiempo; a un $12,4 \%$ se le olvidó y un $12 \%$ tenía miedo.

\section{Discusión}

En tanto el presente estudio tiene un carácter transversal, el mismo no pretende establecer un nexo de causalidad entre los factores analizados y la asistencia al servicio de odontología durante la gestación, sino de asociación entre los mismos. En tal sentido, dado que la medición se realiza simultáneamente, en algunos casos no se pudo determinar la precedencia de un factor con relación a otro, lo que sugiere la existencia de un sesgo de causalidad inversa en algunas asociaciones halladas, como en el caso de recibir orientación sobre salud oral durante el embarazo, la cual puede haber sido recibida al asistir a la consulta de odontología. De igual modo, puede existir un sesgo de memoria, dado el tiempo transcurrido entre la recolección de la información y el evento estudiado, más marcado en aquellas madres que tuvieron problemas durante la gestación. Además, se debe señalar que este estudio no investigó algunos factores del entorno familiar -como el capital social familiar- que puede influir en la decisión de asistencia y en las prácticas en salud oral.

A pesar de estas limitaciones, los resultados del estudio permiten un acercamiento razonable a los determinantes sociales y económicos del acceso a la atención odontológica de mujeres embarazadas en la ciudad de Cali. Así, los principales determinantes sociales encontrados para asistir al odontólogo durante la gestación en Cali están relacionados con aspectos estructurales que reflejan inequidades en salud, como la condición socioeconómica y el nivel educativo alcanzado, y por determinantes intermedios de salud relacionados con los servicios de salud, como el sitio de atención del control prenatal, la remisión a odontología y la orientación en salud oral dada en el control prenatal, junto a factores sociodemográficos como la paridad.

Este es el primer estudio que se lleva a cabo con el objeto de conocer el acceso al servicio dental de la gestante, desde la perspectiva de la madre con hijos recién nacidos en la redes de atención privada y pública de Cali-Colombia. Aunque no se encontró asociación entre las gestantes menores de edad y la asistencia a la consulta odontológica en el análisis multivariado, preocupa el incremento de mujeres adolescentes embarazadas; según estadísticas de la Secretaría de Salud de Cali 17, los partos de las madres con edades entre 10 y 19 años en el año de 2009 representaron el 19\% de todos los partos. Las estadísticas tomadas de la Secretaría de Salud de Cali muestran que en los últimos años ha habido un descenso importante en la fecundidad, pero un incremento en el número de embarazos en las adolescentes. Este estudio encuentra que la mayoría de las menores de edad formaban parte tanto del régimen subsidiado, como del grupo de no aseguradas al sistema de aseguramiento, y que corresponde a los niveles socioeconómicos más bajos de la población, con mayores necesidades básicas insatisfechas. Esta situación puede ser el reflejo de indicadores de pobreza que han aumentado en Cali con cifras que van del $39 \%$ a 67\% entre los años 1998 a 2004 y afecta principalmente a la población joven y a mujeres en edad reproductiva 18. En 2008 el índice de pobreza en Cali fue del 30,1\%, pero subió al 32,6\%, por cuenta de la crisis económica nacional, según un estudio de la Misión para el Empalme de las Series de Empleo, Pobreza y Desigualdad (Mesep) y Planeación Nacional 19.

Esta investigación revela que entre el $80 \%$ y $85 \%$ de las gestantes acuden a la atención odontológica en Cali, cifra muy alta si se compara con los estudios realizados en Latinoamérica 20,21. Es probable que estos resultados favorables de acceso a odontología sea el resultado de las acciones encaminadas en Cali desde el 2008, con la inclusión de la remisión en salud oral de las embarazadas asistentes al control prenatal y el desarrollo de guías de atención en salud oral a la gestante.

Pocos estudios han incluido el estado marital y medido su peso relativo con relación al acceso a la atención odontológica 22,23 . El estar casadas o en unión libre parece tener influencia en la asistencia a la consulta durante la gestación, pues la asociación de este factor con la asistencia a 
consulta resultó estadísticamente significativa, tanto en el análisis bivariado, como al ajustarse con otras variables en el estudio. Esta situación corrobora uno de los hallazgos del estudio realizado por Habashneh et al. 14, en el cual el hecho de tener pareja se identificó como un factor predictor de que las gestantes acudieran al servicio de odontología.

$\mathrm{Al}$ analizarse la condición de ser afrodescendiente, se observó que si bien tres cuartas partes de las mismas acudieron a la consulta odontológica, su acceso fue diferencial con relación a los otros grupos. Este estudio aporta elementos para explorar la oportunidad de acceso de algunos grupos étnicos y es un insumo para identificar en qué medida las desigualdades en salud de las minorías étnicas, con relación a la salud bucodental, son iniquidades.

La escolaridad o nivel de educación ha sido asociada con el acceso al servicio de odontología según estudios previos 21, pero ninguno había presentado el nivel educativo como una variable predictiva de la asistencia de la embarazada a consulta odontológica. Las gestantes que acudieron al servicio público de salud tenían menor nivel de escolaridad que las que fueron a las clínicas privadas, situación similar encontrada en el estudio de Garbero et al. 21. Ahora bien, el hecho de que aproximadamente dos terceras partes de las madres estudiadas tuviera estudios secundarios completos -e incluso algunas con educación superior- representa un hallazgo alentador para la educación en salud oral y la salud general de los bebés que acaban de tener si se tiene en cuenta que la niños siguen en su proceso de aprendizaje las actitudes y comportamiento de la madre 24 .

En este trabajo se analizaron los ingresos a partir de las percepciones de dos aspectos de la vida diaria como la suficiencia de los recursos para cubrir los gastos básicos del hogar y la percepción que tenía sobre la cuantía de sus ingresos la madre entrevistada; además, estas dos variables fueron complemento de la variable tipo de aseguramiento en salud, que es un indicador directo del nivel de clasificación socioeconómica. Se encontró que las gestantes que cuentan con escasos recursos asistieron en menor proporción a la consulta odontológica que las presentaban mejores condiciones, a pesar que el seguro de salud le incluye la atención odontológica en el plan de beneficios. Esta situación es similar a lo encontrado por Lydon-Rochelle et al. ${ }^{25}$ y Habashneh et al. ${ }^{14}$ quienes en sus análisis de regresión simple encontraron asociación significativa del ingreso con la consulta odontológica. En este estudio, a diferencia de las revisiones realizadas, la percepción de suficiencia de recursos para atender ne- cesidades básicas de las gestantes resultó ser una variable predictiva de la asistencia a la consulta odontológica en el modelo final construido.

Asimismo, se encontró que la primiparidad fue un factor asociado con ir a la consulta dental durante la gestación, a diferencia de otros estudios donde sólo se reporta la paridad, pero no la relacionan con la atención odontológica. En este sentido, el desconocimiento de las madres ante un evento nuevo para ellas como la gestación, así como su preocupación por que no hubiera sobresaltos durante el embarazo, pueden haber favorecido su mayor asistencia a la consulta odontológica, a diferencia de las madres más experimentadas.

En esta investigación, el fomento desde el control prenatal para asistir al servicio de odontología durante el embarazo, la remisión dada a la gestante a odontología y la educación en el mismo control prenatal acerca de la salud oral, se identificaron como factores claves para la decisión de la gestante en asistir al servicio. En publicaciones revisadas desde 1990 hasta la fecha, se había encontrado que más de la mitad de las gestantes no recibieron información para asistir al servicio de odontología 25,26, y también que la educación en salud bucodental recibida era un factor significativamente asociadas con la visita de las madres a odontología. Es importante resaltar en este análisis que este estudio muestra como el compromiso del médico y de la enfermera, en el control prenatal en la remisión a la consulta dental, hace que la remisión para valoración odontológica sea la principal causa de consulta en odontología, a diferencia de los estudios realizados a la fecha.

Con relación a la inasistencia a odontología durante la gestación se observa que además de la asociación con bajo nivel socioeconómico, se corrobora hallazgos de otro estudio 27 como el poco interés de asistir, la falta de tiempo y el miedo.

La inclusión de la salud oral como parte de las patologías de interés en salud pública por organismos como Naciones Unidas 28 favorece la atención integral de la gestante afectada por el consumo de tabaco, la diabetes, el embarazo no deseado y el resultado del embarazo, la violencia, los problemas cardiovasculares, la seguridad alimentaria, etc., muchas de las cuales comparten factores comunes de riesgo con patología orales. Aunque las acciones que se centran sólo en el estilo de vida y las influencias conductuales tienen éxito limitado en la reducción de inequidades en salud bucal 29 , el realizar acciones de protección específica y promoción de la salud que tiene un componente conductual no significarían culpar a la víctima, en la medida que podamos transformarla para que haga valer sus derechos y pueda 
hacer uso de sus derechos de ciudadanía para mejorar el ambiente que permita de manera sostenible mantener mejores condiciones para una vida digna.

\section{Resumo}

A fim de determinar a prevalência de atendimento odontológico de gestantes em Cali, Colômbia e identificar fatores socioeconômicos e de serviços de saúde associados com o acesso à atenção odontológico durante a gestação, um estudo transversal foi realizado, no qual foram estudadas 993 mães no pós-parto imediato, que tinham sido tratadas em clínicas públicas e privadas em 2012. Oitenta e três por cento (IC95\%: 80,6-85,4) das mães visitaram o dentista na gravidez. A análise multivariada das variáveis relacionadas ao atendimento odontológico na gestação encontrou associação com os recursos econômicos $(86,6 \%$ suficiência versus 72,5\% insuficiência, OR = 1,8; IC95\%: 1,12,8), o nível de instrução (88\% secundário completo versus $74 \%$ secundário incompleto, $O R=1,7$ IC95\%: 1,1-2,7), a informação brindada na área da saúde oral durante o controle pré-natal e visita ao dentista $(86,4 \%$ sim versus $42,9 \%$ não, OR $=5,7$; IC95\%: 3,110,8). Estes resultados mostram um acesso diferenciado à atenção odontológica entre as gestantes, como aqueles com baixo nível socioeconômico usaram os serviços odontológicos numa menor proporção.

Odontologia em Saúde Pública; Gestantes; Disparidades em Assistência à Saúde; Desigualdades em Saúde
Como conclusión, debe señalarse que a pesar de la buena cobertura en el servicio de odontología, se evidencia la existencia de diferencias sociales injustas y evitables para acceder a la atención dental durante la gestación.

\section{Colaboradores}

J. Corchuelo-Ojeda contribuyó al diseño y ejecución del proyecto, análisis e interpretación de los datos, redacción del artículo, revisión crítica de la importancia del contenido intelectual y aprobación de la versión final. G. J. González Pérez colaboró en el análisis de los datos y la redacción del artículo.

\section{Agradecimientos}

Los autores agradecen a la Secretaría de Salud de Cali el apoyo logístico en la presentación del proyecto en la Redes públicas y privadas de Salud. A la psicóloga Sandra Horta por su orientación al equipo para estandarización de las entrevistas. 


\section{Referencias}

1. Lopez NJ, Smith PC, Gutierrez J. Higher risk of preterm birth and low birth weight in women with periodontal disease. J Dent Res 2002; 81:58-63.

2. Boggess K, Madianos P, Preisser J, Moise K, Offenbacher S. Chronic maternal and fetal Porphyromonas gingivalis exposure during pregnancy in rabbits. Am J Obstet Gynecol 2005; 192:554-7.

3. Sanders A, Spencer A, Slade G. Evaluating the role of dental behaviour in oral health inequalities. Community Dent Oral Epidemiol 2006; 34:71-9.

4. Ferreira FV, Gasparin AB, Soeiro F, Oliveira MDM, Praetzel JR. Percepção de médicos obstetras sobre a saúde bucal de gestantes. Int J Dent 2009; 8:72-8.

5. Gonçalves J, Knupp R. Avaliação do conhecimento dos pediatras sobre saúde oral na primeira infancia. J Bras Odontopedriatr Odontol Bebê 2000; 3 : 496-9.

6. Moimaz S, Rocha N, Saliba O, Garbin C. O acesso de gestantes ao tratamento odontológico. Rev Odontol Univ Cid São Paulo 2007; 19:39-45.

7. Rossel SL, Mayberry LJ. Pregnancy and oral health: a review and recommendations to reduce gaps in practice and research. MCN Am J Matern Child Nurs 2008; 33:32-7.

8. Lopez NJ, Smith PC, Gutierrez J. Periodontal therapy may reduce the risk of preterm low birth weight in women with periodontal disease: a randomized controlled trial. J Periodontol 2002; 73:911-24.

9. Lopez N, Da S, Ipinza J, Gutierrez J. Periodontal therapy reduces the rate of preterm low birth weight in women with pregnancy-associated gingivitis. J Periodontol 2005; 76:2144-53.

10. Corchuelo J. A literature review on social and economic factors related to access to dental care for pregnant women. The Journal of Dentists 2013; 1:24-34.

11. Departamento Administrativo de Planeación. Cobertura en salud según SISBÉN, FOSIGA Y DANE: Subregión Sur del Valle del Cauca, 2008 - 2012 [PDF]. Valle del Cauca: Departamento Administrativo de Planeación; 2013. (Boletines Socioeconómicos Subregión Sur, 5).

12. Secretaría de Salud de Cali. Informe de ejecutivo de gestión año 2010. Programa Ampliado de Inmunizaciones PAI. http://calisaludable.cali.gov. co/saludPublica/2011_PAI/InformeGestion2010/ GESTION_ANIO_2010.pdf (accedido el 10/May/ 2011)

13. Corchuelo J. Factores sociodemográficos relacionados con la historia de caries y nivel de placa bacteriana en gestantes y mujeres en edad reproductiva en el Valle del Cauca. Cali: Fundación Ceges; 2010.

14. Habashneh R, Guthmiller J, Levy S, Johnson G, Squier C, Dawson D, et al. Factors related to utilization of dental services during pregnancy. J Clin Periodontol 2005; 32:815-21.

15. Strafford K, Shellhaas C, Hade E. Provider and patient perceptions about dental care during pregnancy. J Matern Fetal Med 2008; 21:63-71.
16. Ministerio de Salud. III Estudio Nacional de Salud bucal ENSAB III. Santa Fé de Bogotá: Ministerio de Salud; 1999.

17. Secretaría de Salud de Cali. Tabla de contenido. http://calisaludable.cali.gov.co/proyectos/ Informe_Plan_de_Desarrollo_2009/informe_gestion_plan_2009.pdf (accedido el 12/Nov/2012).

18. Centro para el Desarrollo y Evaluación de Políticas y Tecnología en Salud Pública, Universidad del Valle. Situación de salud de Cali: una mirada desde la salud pública. http://www.cedetes.org/modelo/ files/SITUACION_DE_SALUD_DE_CALI.pdf (accedido el 20/Oct/2012).

19. El País. Estudio revela que Cali ha reducido sus indicadores de pobreza. http://www.elpais.com. co/elpais/cali/noticias/estudio-revela-cali-hareducido-sus-indicadores-pobreza (accedido el 05/Nov/2012).

20. Vieira G, Zocratto K. Percepção das gestantes quanto a sua saúde bucal. RFO UPF 2007; 12:27-31.

21. Garbero I, Delgado A, Benito de Cárdenas I. Oral health in pregnant: knowledge and attitudes. Acta Odontol Venez 2005; 43:135-40.

22. Dooley S. Perceived health needs and receipt of services during pregnancy - Oklahoma and South Carolina. MMWR Morb Mortal Wkly Rep 2010; 59:710-4.

23. Timothe P, Eke P, Presson S, Malvitz D. Dental care use among pregnant women in the United States reported in 1999 and 2002. Prev Chronic Dis 2005; 2(1). http://www.cdc.gov/pcd/issues/2005/ jan/04_0069.htm

24. Thomas JF, Startup R. Some social correlates with the dental health of young children. Community Dent Health 1992; 9:11-7.

25. Lydon-Rochelle M, Krakowiak P, Hujoel P, Peters R. Dental care use and self-reported dental problems in relation to pregnancy. Am J Public Health 2004; 94:765-71.

26. Saddki N, Yusoff A, Yew H. Factors associated with dental visit and barriers to utilisation of oral health care services in a sample of antenatal mothers in Hospital Universiti Sains Malaysia. BMC Public Health 2010; 10:75.

27. Scavuzzi I, Rocha M, Vianna M. Percepção sobre atenção odontológica na gravidez. JBP, J. Bras Odontopediatr Odontol Bebê 1998; 1:43-50.

28. Petersen P, Kwan S. Equity, social determinants and public health programmes - the case of oral health. Community Dent Oral Epidemiol 2011; 39:481-7.

29. Watt RG. From victim blaming to upstream action: tackling the social determinants of oral health inequalities. Community Dent Oral Epidemiol 2007; 35:1-11.

Recibido el 21/Ago/2013

Versión final presentada el 18/Mar/2014

Aprobado el 27/Mar/2014 